\title{
Management of Diaphragmatic Injury during Transperitoneal Laparoscopic Urological Procedures
}

\author{
Octavio A. Castillo, Gonzalo Vitagliano, Mauricio Moreno, Manuel A. Diaz, Oscar Cortes \\ Department of Urology (OAC, GV, MM, MAD, OC), Clinica Santa Maria, and Department of \\ Urology (OAC), School of Medicine, Universidad de Chile, Santiago de Chile, Chile
}

\begin{abstract}
Introduction: Carbon dioxide pneumothorax is a rare complication in laparoscopic urology, but with the widespread use of laparoscopy and the increasing surgical pathologies managed by this technique this infrequent complication has become a potential risk.

Materials and Methods: A total of 786 laparoscopic transperitoneal urologic operations were reviewed at our institution. All procedures were performed by the same surgeon and included 213 adrenalectomies, 181 simple nephrectomies, 143 lymphadenectomies, 118 radical nephrectomies, 107 partial nephrectomies and 24 nephroureterectomies. Mean patient age was 53.2 years (range 24 to 70). Mean BMI was $28.15 \mathrm{Kg} / \mathrm{m}^{2}$ (range 20 to 48.9).

Results: A total of 6 cases $(0.7 \%)$ of diaphragmatic injury were found. All reported patients had additional factors that may have contributed to diaphragmatic injury. Diaphragmatic repair was always carried out by intracorporeal suturing and only one case required chest tube placement. All patients evolved uneventfully.

Conclusions: Repair of diaphragmatic injuries should always be attempted with intracorporeal suture since this is a feasible, reproducible and reliable technique.
\end{abstract}

Key words: pneumothorax; diaphragm; intraoperative complications; laparoscopy Int Braz J Urol. 2007; 33: 323-9

\section{INTRODUCTION}

The clear advantages that laparoscopy provides over open surgery have made this technique very popular in the last decade. However, for urologist laparoscopy has gained wide acceptance only in recent years. Inadvertent diaphragmatic injury during transperitoneal laparoscopic surgery in urology is a rare occurrence (1). Yet, with the widespread use of laparoscopy and the increasing surgical pathologies managed with this technique, there is a potential for an increased risk of carbon dioxide pneumothorax due to diaphragmatic injury. Capnothorax associated with laparoscopic surgery is different from air pneumothorax and may call for a different treatment.

We report the laparoscopic management of six cases with iatrogenic diaphragmatic injury during laparoscopic urologic surgery.

\section{MATERIALS AND METHODS}

The records of 786 laparoscopic transperitoneal urologic operations were reviewed. All 
procedures were performed by the same surgeon (OAC) and included: 213 adrenalectomies, 181 simple nephrectomies, 143 retroperitoneal lymphadenectomies, 118 radical nephrectomies, 107 partial nephrectomies for cancer and 24 radical nephroureterectomies. A total of 6 cases of diaphragmatic injury were found. Mean patient age was 53.2 years (range 24 to 70). Mean BMI was 28.15 (range 20 to 48.9). Operative and clinical records were reviewed and patient outcome evaluated. Details of injury identification and operative management are specified (Table-1).

For diaphragmatic repair, no additional trocars were needed. In most cases, usual working port configuration for adrenalectomy and nephrectomy allowed intracorporeal suturing. However, if necessary an additional $5 \mathrm{~mm}$ port was placed on left or right flank respectively. The defect was repaired with separate 0 -poliglactine sutures while pneumoperitoneum was decreased in $12 \mathrm{mmHg}$. Before the stitches were secured, the anesthesiologist administered a large inspiratory breath (Figures-1 and 2).

Criteria used for chest tube placement was pneumothorax greater than $20 \%$ of lung volume or associated with hemodynamic or ventilatory changes.

\section{RESULTS}

A total of 6 cases of diaphragmatic injury were recorded during three adrenalectomies, one partial nephrectomy, one radical nephrectomy and one left retroperitoneal lymph node dissection respectively. In all cases, the cause of pleural lesion was iatrogenic injury to the diaphragm.

Case 1 - A 24-year-old woman (BMI 21) with a history of ulcerative colitis and an incidental $12 \mathrm{~cm}$ left adrenal cyst underwent laparoscopic adrenalectomy. During surgery an increase in end inspiratory pressure and end tidal carbon dioxide levels was noted. This prompted the inspection of the operative field. A $2 \mathrm{~cm}$ lesion was found on the left diaphragm, no pulmonary parenchyma was evident. The defect was repaired with 0-poliglactine sutures while pneumoperitoneum was decreased in $12 \mathrm{mmHg}$ with the anesthesiologist administering a large inspiratory breath before securing the stitches. Adrenalectomy was com-

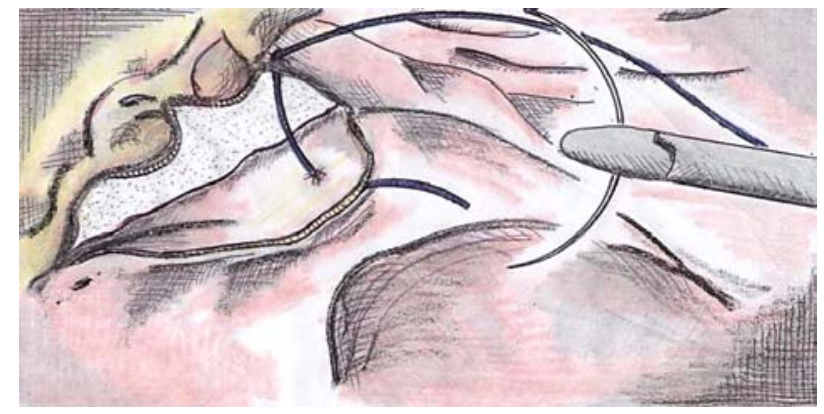

Figure 1 - Diaphragmatic lesion repaired with intracorporeal suturing.

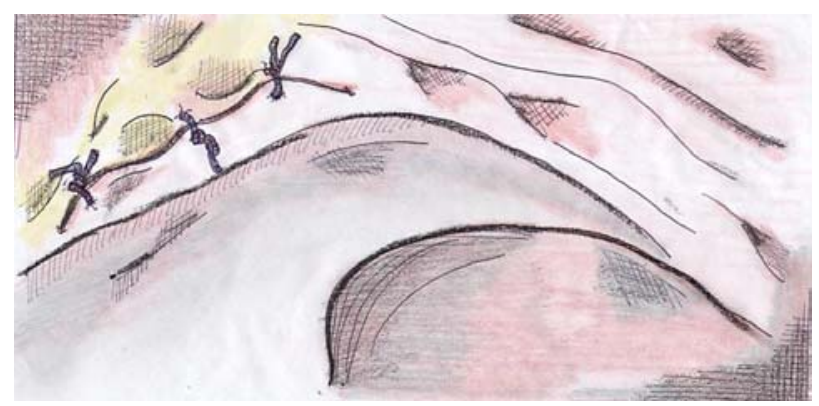

Figure 2 - Diaphragmatic repair completed.

pleted with a total time of 135 minutes. A chest tube was placed and left for 12 hours. Follow-up chest $\mathrm{x}$ ray showed no residual pneumothorax. The patient was discharged home after 48 hours.

Case 2 - A 40-year-old woman (BMI 24.5) underwent a right adrenalectomy for an incidental 7 $\mathrm{cm}$ right adrenal mass. During adrenal dissection a 3 $\mathrm{cm}$ iatrogenic injury was identified on the right diaphragm with pulmonary parenchyma exposure. The pleural cavity was inspected with the laparoscope and a pleural mass was observed. A biopsy specimen was taken and the diaphragmatic defect was repaired using the technique previously described. The procedure was finished in a total of 60 minutes. Follow-up $\mathrm{x}$-rays showed no residual pneumothorax. Final histologic examination revealed an adrenal adenoma and a pleural teratoma. The patient was discharged home after 48 hours.

Case 3 - A 70-year-old female patient (BMI 48.9 ) with a $3 \mathrm{~cm}$ lateral right mid pole renal tumor underwent a partial nephrectomy. The partial nephrectomy was carried out under warm isquemia of $25 \mathrm{~min}$ - 


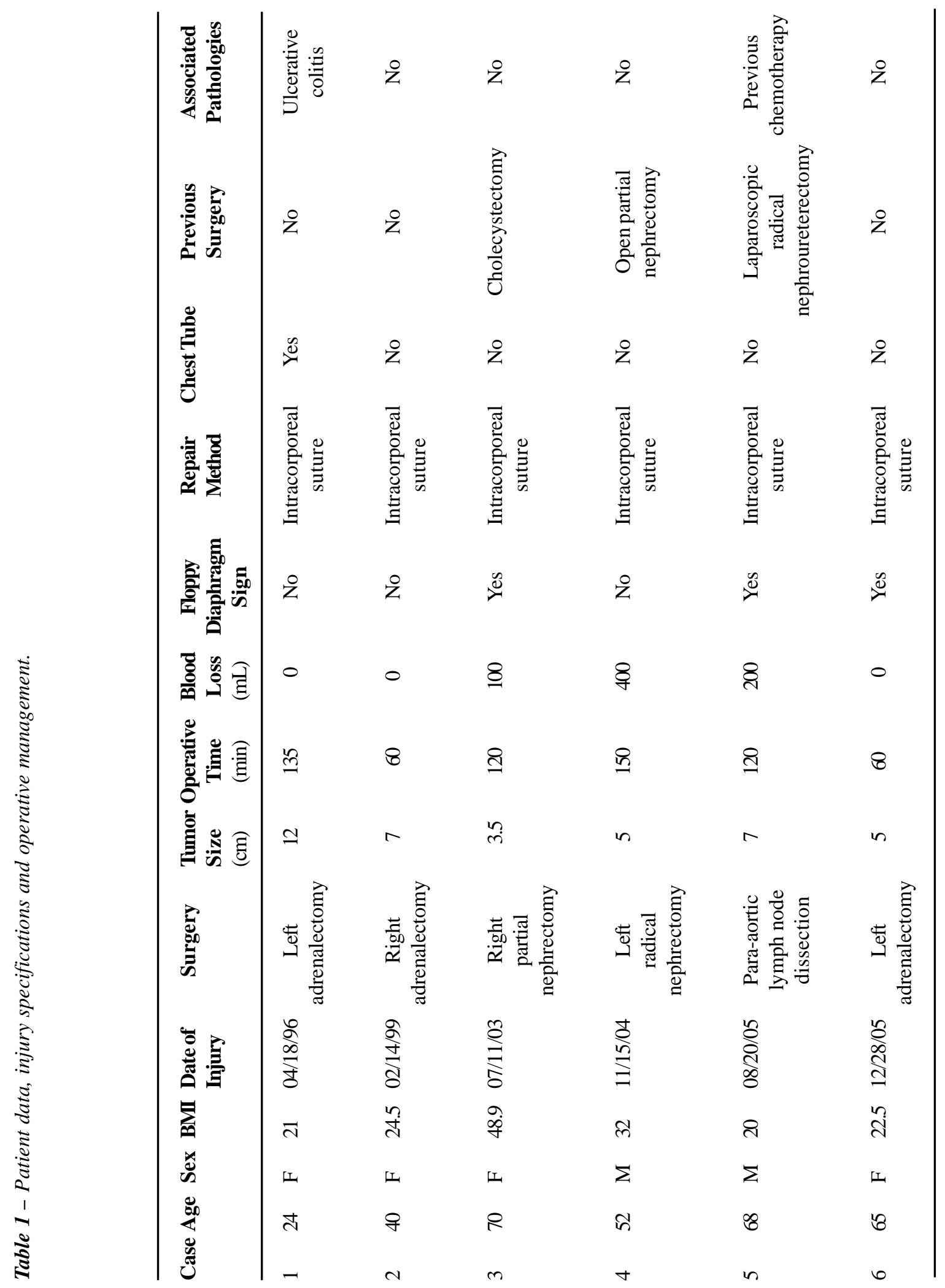


utes with a total operative time of 120 minutes. While the upper pole of the right kidney was being dissected the "floppy diaphragm" sign was observed. After inspection a $1 \mathrm{~cm}$ diaphragmatic lesion was evident. No pulmonary parenchyma was seen. The defect was repaired with the technique described above. There was no need for tube thoracostomy and follow-up x-rays revealed complete resolution of the pneumothorax. The patient was discharged after 48 hours.

Case 4 - A 52-year-old man (BMI 32) that underwent a previous open left partial nephrectomy for renal carcinoma was submitted to a laparoscopic left radical nephrectomy for a $5 \mathrm{~cm}$ recurrent tumor. During surgery a $4 \mathrm{~cm}$ diaphragmatic injury was observed and repaired laparoscopically as described before. The procedure was finished in 150 minutes. There was no need for chest tube and the patient was discharged on postoperative day 5 .

Case 5 - A 68-year-old male patient (BMI 20) with a history of laparoscopic left radical nephroureterectomy and previous chemotherapy underwent a left retroperitoneal lymph node dissection for a $7 \mathrm{~cm}$ left para-aortic mass. During surgery a 3 $\mathrm{cm}$ diaphragmatic defect was evidenced in association with the sudden inferior billowing of the diaphragmatic wall. The defect was repaired as previously described. The procedure was completed in $120 \mathrm{~min}$ utes. There was no need for tube thoracostomy and follow-up x-rays showed no residual pneumothorax. The patient was discharged on postoperative day 3 .

Case 6 - A 65-year-old woman (BMI 22.5) underwent a left adrenalectomy for an incidental 5 $\mathrm{cm}$ left adrenal mass diagnosed during lung cancer staging. During the procedure two $1 \mathrm{~cm}$ diaphragmatic lesions were produced while the peritoneum was dissected over the colon. The "floppy diaphragm" sign was unmistakable in this case. The lesions were repaired and the procedure was finished in a total of 60 minutes. No chest tube was put in place and followup x-rays showed no residual pneumothorax. The patient was discharged on postoperative day 3 .

\section{COMMENTS}

Iatrogenic injury of the diaphragm during general laparoscopy is unusual. In laparoscopic renal and adrenal surgery this complication does not exceed $0.6 \%$ in the largest series (2). The occasional occurrence of this complication is due to the clear separation that exists between the kidneys and the diaphragm (1). However, with the advances made in laparoscopic renal and adrenal surgery, more surgeons are expanding the limits for laparoscopy by attempting very demanding procedures. This may sustain or even increase the incidence of iatrogenic diaphragmatic injuries. It is noteworthy that this series reflects the experience of a single surgeon that has surpassed the learning curve of standardized techniques (OAC).

All of the patients that we report have additional factors that may have lead to diaphragmatic injury. Morbid obesity, large tumors, inflammatory intestinal pathologies, previous surgeries and chemotherapy are some of the factors that can facilitate the occurrence of diaphragmatic lesions. However, adrenal surgery by itself has an inherent risk for diaphragmatic injury because the adrenal gland is juxtaposed against the diaphragm. Table-1 summarizes patient data, injury specifications and operative management.

Diaphragmatic injury can originate from improper trocar placement or direct contact with monopolar electrocautery or harmonic scalpel (2). When the retroperitoneal approach is preferred for renal or adrenal surgery improper trocar placement can easily lead to diaphragm injury (2).

The lesion can appear as an evident tear of the diaphragm or be invisible to the surgeon's inspection and be alerted by changes in patient cardiopulmonary status. Pneumothorax may produce changes in auscultation, end inspiratory pressure, blood pressure and arterial blood gasses (3). In addition, an undetected injury may become evident by the floppy diaphragm sign, in which the diaphragm billows inferior with any degree of abdominal desufflation, reflecting the loss of negative pressure within the diaphragm (4). In our series, this sign was evident in 3 out of the 6 patients and prompted the rapid diagnosis of the diaphragmatic injury. We also report a case in which pleural cavity inspection was done with the laparoscope through the diaphragmatic lesion in order to perform a biopsy of a suspicious pleural area that was evident after the injury took place. This suspicious area later proved to be pleural teratoma. 
The anesthesiologist involvement is decisive in the diagnosis and timing of repair. Carbon dioxide pneumothorax may go undetected intraoperatively and close monitoring of cardiopulmonary status may alert of the injury.

In order to avoid diaphragmatic injury, care must be taken when large adrenal masses are dissected and during the mobilization of intra-abdominal structures for kidney exposure.

Our report is the largest series after the multicentric work published by Del Pizzo et al. of the New York Presbyterian Hospital (2). Similar to what was described by Del Pizzo we also chose interrupted polyglactin sutures for the laparoscopic repair regardless of lesion size and location. Previous reports show that multiple techniques can be used to repair the diaphragm. In one specific case of a handassisted nephrectomy, the author chose to leave a dual layer mesh of polypropylene and polyglactin for a $1 \mathrm{~cm}$ lesion. The mesh was secured to the diaphragmatic rent by aid of a laparoscopic stapler and the surgeon's hand (5). Several reports confirm the feasibility of diaphragmatic repair by means of intracorporeal suturing $(2,3,6)$. We believe that diaphragm suturing must always be attempted due to the simplicity and reliability of this technique. Nevertheless there has been one successful report of diaphragmatic injury repair without the use of stitches (7). This was achieved by employing a matrix gel and a thrombin solution (Floseal ${ }^{\circledR}$ ) with interposition of the omentum over a $1 \mathrm{~cm}$ diaphragmatic lesion. The authors refer to their technique as a suitable option for small lesions. To reach an effective repair of the diaphragm, air must be evacuated before the stitches are secured by means of either a suction device or the administration of a long forced inspiratory breath. In addition, repair of diaphragmatic injury has to be timed according to patient parameters and feasibility of repair. When the patient is in stable condition surgery can continue and the injury may be addressed at the end of the procedure. In cases of large tumors that may obstruct the surgeon's direct access to the lesion, surgical specimen should be removed first in order to ease repair. Nevertheless we think that if possible, the diaphragm injury should be repaired without delay. This was the case in all of our patients in which early recognition of diaphragmatic injury allowed for a prompt repair without the interference of the surgery. Pneumothorax greater than $20 \%$ of lung volume or associated with hemodynamic or ventilatory changes is managed with thoracostomy (6). Pleural lesions produced by trocar placement or important residual capnothorax may also warrant thoracostomy. Compared to air, carbon dioxide has higher solubility and increased diffusion coefficient, this allows a greater amount of molecules to diffuse across a membrane in a given time. This explains why capnothorax usually resolves spontaneously and allows for expectant management in stable patients (6).

In case $n^{\circ} 1$ ventilatory changes were evident with carbon dioxide retention, but the patient remained hemodynamically stable, the lack of experience in the management of capnothorax prompted a chest tube placement. Retrospectively we think this could have been avoided. Abreu et al., reported a higher incidence of gas collections associated with the retroperitoneal over the transperitoneal approach $(6.6 \%$ vs. $0.7 \%)(8)$. However, they concluded that asymptomatic, subclinical, spontaneously resolving gas collections in the chest are more common with retroperitoneoscopy but the incidence of symptomatic or serious thoracic complications is similar between transperitoneal and retroperitoneal laparoscopy (9). We did not observe injuries from direct trocar entry in our series; this can be explained by the fact that we prefer the transperitoneal to the retroperitoneal approach for renal or adrenal surgery.

\section{CONCLUSIONS}

While uncommon, iatrogenic injury of the diaphragm is a possible complication of laparoscopic transperitoneal urologic procedures. Patient characteristics, adrenal surgery and tumor size may be associated with a higher risk of injury. Care must be taken to prevent it and surgeons need to be trained to resolve it. Repair of diaphragmatic injuries should always be attempted with intracorporeal suture since this is a feasible, reproducible and reliable technique. 


\section{CONFLICT OF INTEREST}

None declared.

\section{REFERENCES}

1. Vallancien G, Cathelineau X, Baumert H, Doublet JD, Guillonneau B: Complications of transperitoneal laparoscopic surgery in urology: review of 1,311 procedures at a single center. J Urol. 2002; 168: 23-6.

2. Del Pizzo JJ, Jacobs SC, Bishoff JT, Kavoussi LR, Jarrett TW: Pleural injury during laparoscopic renal surgery: early recognition and management. J Urol. 2003; 169: 41-4.

3. Potter SR, Kavoussi LR, Jackman SV: Management of diaphragmatic injury during laparoscopic nephrectomy. J Urol. 2001; 165: 1203-4.

4. Voyles CR, Madden B: The "floppy diaphragm" sign with laparoscopic-associated pneumothorax. JSLS. 1998;2:71-3.

Correspondence address:

Dr. Octavio Castillo

Av Santa Maria 500

Providencia, Santiago, Chile

Fax:0056-2461-2875

E-mail: octaviocastillo@vtr.net

\section{EDITORIAL COMMENT}

Inadvertent diaphragmatic injury is a rare, but a serious matter for laparoscopic surgery of the upper urinary tract. On the one hand, as stated by the authors, aggravating circumstances such as obesity, large tumors, or previous surgery may increase the risk of an inadvertent injury; on the other hand, lack of experience and technical errors can enforce this complication. Whereas major surgical difficulties are
5. Gonzalez CM, Batler RA, Feldman M, Rubenstein JN, Nadler RB, Schoor RA: Repair of a diaphragmatic injury during hand assisted laparoscopic nephrectomy using an onlay patch of polypropylene and polyglactin mesh. J Urol. 2002; 167: 2512-3.

6. Venkatesh R, Kibel AS, Lee D, Rehman J, Landman J: Rapid resolution of carbon dioxide pneumothorax (capno-thorax) resulting from diaphragmatic injury during laparoscopic nephrectomy. J Urol. 2002; 167: 1387-8.

7. Bhayani SB, Grubb RL 3rd, Andriole GL: Use of gelatin matrix to rapidly repair diaphragmatic injury during laparoscopy. Urology. 2002; 60: 514.

8. Abreu SC, Sharp DS, Ramani AP, Steinberg AP, Ng CS, Desai MM, et al.: Thoracic complications during urological laparoscopy. J Urol. 2004; 171: 1451-5.

9. Shanberg AM, Zagnoev M, Clougherty TP:.Tension pneumothorax caused by the argon beam coagulator during laparoscopic partial nephrectomy. J Urol. 2002; 168: 2162 .

Accepted after revision: January 31, 2007

best managed by extensive experience of the surgeon, technical errors can be avoided by paying attention to a few guidelines.

During retroperitoneoscopy, a diaphragmatic injury (caused by improper trocar placement) is usually avoided by an initial lumbodorsal incision within the muscle-free triangle between latissimus dorsi muscle and oblique external muscle. Using this ac- 
cess technique the following trocars, in particular the upper ones, are placed under palpatory control, therefore usually avoiding injuries to the diaphragm (1).

Due to the increasing numbers of laparoscopic centers worldwide, special care has to be taken to train the individual surgeon. In our opinion and as stated previously, every surgeon should run through a training program prior to the clinical setting, which imparts the basics of laparoscopic intracorporeal suturing and knotting techniques. Only thereafter is it possible to ensure that a quick and safe management of complications can be provided $(2,3)$.

\section{REFERENCES}

1. Rassweiler J, Seemann O, Frede T, Henkel T, Alken P: Retroperitoneoscopy: experience with 200 cases. J Urol. 1998; 160: 1265-9.

2. Teber D, Dekel Y, Frede T, Klein J, Rassweiler J: The Heilbronn laparoscopic training program for laparoscopic suturing: concept and validation. $\mathbf{J}$ Endourol. 2005; 19: 230-8.

3. Rassweiler J, Klein J, Teber D, Schulze M, Frede T: Mechanical simulators for training for laparoscopic surgery in urology. J Endourol. 2007; 21: 252-62.

Dr. Thomas Frede

Chair, Department of Urology Helios-Klinik Müllheim

Müllheim, Germany

E-mail:Thomas.frede@helios-kliniken.de 